\title{
Comunicación

\section{La construcción del mercado audiovisual europeo: balance de dos décadas de políticas públicas}

The construction of the European audiovisual market:

Assessing two decades of public policy

El programa europeo de apoyo a la industria audiovisual (Media) ha cumplido 20 años de existencia (19912011). Este artículo tiene como objetivo analizar la evolución de esta política hacia la internacionalización. El programa Media ha buscado nuevos socios y mercados en el entorno iberoamericano e internacional; el análisis también evalúa su impacto interno en el espacio audiovisual europeo.

PALABRAS ClAVE: Unión Europea, política audiovisual europea, programa Media, industria audiovisual.
The EU's support program for the European audiovisual industry (Media) has recently celebrated its 20th anniversary (1991-2011). This article analyzes its evolution towards internationalization. Against the background of media's search for new partners and markets in the Iberoamerican and international landscape; this analysis assesses the program's impact on the European audiovisual space.

KEY WORDS: European Union, European Audiovisual Policy, Media program, audiovisual industry.

1 Universidad Autónoma de Barcelona, España.

Correo electrónico: carmina.crusafon@uab.cat

Campus de Bellaterra, Edificio I, C.P. 08193; Barcelona, España. 


\section{INTRODUCCIÓN}

El proceso de integración regional en Europa presenta un grado de desarrollo notable, fruto de una trayectoria que se inicia en 1957 con la firma del Tratado de Roma. Durante estas cinco décadas, el proceso europeo ha pasado por diversas fases: desde un inicio como Comunidad Europea del Carbón y el Acero, pasando por la Comunidad Económica Europea (CEE), hasta la constitución de la unión política y económica, que constituye la forma actual de la Unión Europea (UE). ${ }^{2}$

Fruto del avance del proyecto europeo, la dimensión supraestatal ha ido tomando mayor protagonismo y las competencias que dependen de las instituciones comunitarias cada vez son más numerosas. Como consecuencia, las políticas públicas europeas se convierten en los principales ejes de actuación en muchos de los sectores de la economía.

Un ejemplo del protagonismo de la dimensión europea lo tenemos en el sector audiovisual. En términos de mercado, esta industria genera un volumen de ingresos de 107,400 millones de euros y supone $1.2 \mathrm{mi}-$ llones de puestos de trabajo altamente calificados. Además forma parte de lo que se conoce como la industria cultural y creativa que equivale a 4.5\% del Producto Interno Bruto (PIB) europeo. En términos de cultura, el audiovisual europeo es el embajador de la identidad y cultura europea, así como una de las vías de expresión de la manera de entender el mundo y la realidad que se tiene en el viejo continente. Es por su naturaleza cultural, que el audiovisual fue incluido por primera vez en el articulado de un tratado europeo, precisamente fue dentro del Tratado de Maastricht en su artículo 128,3 en 1993.

La necesidad de consolidar un espacio audiovisual común, en consonancia con la creación de un mercado europeo y como parte esencial

2 La UE es una asociación económica y política única, formada por 27 países europeos: Alemania, Austria, Bélgica, Bulgaria, Chipre, Dinamarca, Eslovaquia, Eslovenia, España, Estonia, Finlandia, Francia, Grecia, Holanda, Hungría, Inglaterra, Irlanda, Italia, Letonia, Lituania, Luxemburgo, Malta, Polonia, Portugal, República Checa, Rumania y Suecia.

3 Este artículo es en la actualidad el 167 del Tratado Final de la Unión Europea (TFEU). 
del proceso de integración regional, está en la base del desarrollo de la Política Audiovisual Europea (PAE), cuyos inicios se sitúan a finales de la década de los ochenta. Es precisamente en este marco donde se ubica el programa europeo de apoyo a la industria audiovisual, conocido bajo las siglas MEDIA. ${ }^{4}$ Este instrumento comunitario constituye uno de los pilares de la PAE, junto con la Directiva de Servicios de Comunicación Audiovisual. ${ }^{5}$ El programa MEDIA tiene sus inicios en el año 1991 y ahora cumple su 20 aniversario. Durante este tiempo, el programa ha pasado por diferentes ediciones y ha invertido un total de 1,780 millones de euros. En la actualidad, la mitad de las películas europeas que podemos ver en los cines cuentan con el apoyo del MEDIA. Así mismo el MEDIA ha sufrido una gran evolución, no solo en los países que participan en él, que hoy llegan a 32 (véase Tabla 1), sino también desde su internacionalización, ya que desde 2008 ha incorporado una modalidad de ayudas para proyectos con terceros países. Además, este programa ha tenido diferentes prioridades en función de las directrices establecidas por la Comisión Europea (CE). 6 Esto es, según el periodo se ha potenciado la dimensión industrial y/o cultural, fruto de la naturaleza dual del audiovisual.

Para analizar la evolución de dicho programa, este artículo se estructura en tres partes. La primera presenta la evolución del programa MEDIA en sus distintas ediciones, señalando sus aspectos más importantes (objetivos, acciones, y presupuesto); la segunda se enmarca en un análisis de cómo ha evolucionado el programa hacia la internacionalización y su impacto interno en la construcción del espacio audiovisual europeo; y la tercera se centra en señalar los retos a los que se enfrenta

$4 \quad$ Las siglas de este programa responden a los conceptos en francés: Mesures d'Encouragement De l'Industrie Audiovisuel (MEDIA). Esto es, medidas de fomento para la industria audiovisual.

5 La Directiva de Servicios de Comunicación Audiovisual 2007/65/EC es la gran norma legal europea que rige en el sector audiovisual en todos los países miembros de la UE. Esta Directiva es la versión actualizada de la Directiva "Televisión sin Fronteras" (aprobada en 1989 y actualizada en 1997).

6 La CE representa y defiende los intereses del conjunto de la UE, elabora propuestas de nueva legislación europea y gestiona la labor cotidiana de poner en práctica las políticas y hacer uso de los fondos europeos. 
este programa dentro del objetivo general de la UE para el año 2020.7 La metodología utilizada es el resultado de la revisión de los documentos oficiales de la UE, así como de artículos y bibliografía académica, que a su vez ha sido completada por entrevistas realizadas a profesionales del sector y a funcionarios de CE.

\section{LA EVOLUCIÓN DEL PROGRAMA MEDIA: LOS EJES DE LA ACTUACIÓN COMUNITARIA}

Durante sus 20 años de existencia, el programa MEDIA (CE, s.f.-b) cuenta con cuatro ediciones y ha ampliado el número de los países participantes, de los 12 iniciales hasta los 32 actuales. Como muestra la Tabla 1, el programa ha seguido la misma evolución que la propia UE en sus diferentes ampliaciones. Cada una de las ediciones ha tenido una duración de cinco años, aunque la edición actual (2007-2013) se ha ampliado hasta siete. Así mismo el programa se ha internacionalizado como resultado de la necesidad de la industria europea de estar presente en el mercado global. En primer lugar, se hizo una experiencia piloto, conocida como MEDIA INTERNATIONAL, entre 2008 y 2010, y posteriormente se aprobó el programa MEDIA MUNDUS para el periodo 2011-2013. De esta manera, la UE puede dar apoyo a proyectos audiovisuales que sean el resultado de la colaboración entre los estados miembros y terceros países. Otro de los elementos en incremento ha sido el presupuesto, que pasó de los 230 millones de ecus 8 en 1991, a los 755 millones de euros para el periodo 2007-2013.

7 Europa 2020 es la estrategia de crecimiento de la UE para la próxima década. Concretamente, la UE ha establecido para el año 2020 cinco ambiciosos objetivos en materia de empleo, innovación, educación, integración social y clima/energía. En cada una de estas áreas, cada Estado miembro se ha fijado sus propios objetivos. La estrategia se apoya en medidas concretas tanto de la UE como de los Estados miembros (CE, s.f.-a)

8 El ECU (European Currency Unit) era la moneda europea que se utilizaba en los ámbitos comunitarios antes de la aprobación del euro en el año 2000. $\mathrm{Su}$ valor respondía a una cesta de monedas de los distintos países miembros de la CE. 


\section{TABLA 1}

EVOLUCIÓN DEL PROGRAMA MEDIA

\begin{tabular}{|c|c|c|c|c|}
\hline Programa & Año & Países participantes & $\begin{array}{l}\text { Total de } \\
\text { países }\end{array}$ & Presupuesto \\
\hline \multirow[t]{3}{*}{$\begin{array}{l}\text { MEDIA I } \\
(1991-1995)\end{array}$} & 1991 & $\begin{array}{l}\text { Alemania, Bélgica, } \\
\text { Dinamarca, España, Grecia, } \\
\text { Francia, Irlanda, Italia, } \\
\text { Luxemburgo, Holanda, } \\
\text { Portugal e Inglaterra }\end{array}$ & 12 & $\begin{array}{l}230 \text { millones } \\
\text { de ecus }\end{array}$ \\
\hline & 1992 & $\begin{array}{l}\text { Islandia, Lichtenstein, } \\
\text { Noruega }\end{array}$ & 15 & \\
\hline & 1995 & Austria, Suecia, Finlandia & 18 & \\
\hline $\begin{array}{l}\text { MEDIA II } \\
(1996-2000)\end{array}$ & & Malta & 19 & $\begin{array}{l}310 \text { millones } \\
\text { de ecus }\end{array}$ \\
\hline \multirow[t]{3}{*}{$\begin{array}{l}\text { MEDIA Plus } \\
(2001-2006)\end{array}$} & 2002 & $\begin{array}{l}\text { Polonia, Letonia, Estonia, } \\
\text { República Checa, Bulgaria } \\
\text { y Lituania }\end{array}$ & 25 & $\begin{array}{l}400 \text { millones } \\
\text { de euros }\end{array}$ \\
\hline & 2003 & $\begin{array}{l}\text { Hungría, Eslovaquia, Chipre } \\
\text { y Eslovenia }\end{array}$ & 29 & \\
\hline & 2006 & Suiza & 30 & \\
\hline \multirow[t]{3}{*}{$\begin{array}{l}\text { MEDIA } 2007 \\
(2007-2013)\end{array}$} & 2007 & Rumania & 31 & $\begin{array}{l}755 \text { millones } \\
\text { de euros }\end{array}$ \\
\hline & 2009 & Croacia & 32 & \\
\hline & & $\begin{array}{l}\text { Otros países en } \\
\text { negociaciones } \\
\text { (véase Tabla 3) }\end{array}$ & & \\
\hline $\begin{array}{l}\text { MEDIA } \\
\text { INTERNATIONAL } \\
(2008-2010)\end{array}$ & 2008 & \multicolumn{2}{|c|}{$\begin{array}{l}\text { Países participantes en el MEDIA + } \\
\text { terceros países }\end{array}$} & $\begin{array}{l}10 \text { millones } \\
\text { de euros }\end{array}$ \\
\hline $\begin{array}{l}\text { MEDIA } \\
\text { MUNDUS } \\
(2011-2013) \\
\end{array}$ & 2011 & \multicolumn{2}{|c|}{$\begin{array}{l}\text { Países participantes en el MEDIA + } \\
\text { terceros países }\end{array}$} & $\begin{array}{l}15 \text { millones } \\
\text { de euros }\end{array}$ \\
\hline
\end{tabular}

Fuente: Elaboración propia con base en datos de la CE (s.f.-b) y Euréval (2007, noviembre). 
Este epígrafe realiza un recorrido por cada una de las ediciones, señalando sus líneas de acción y sus principales resultados. El objetivo final es el de presentar cómo el programa MEDIA se ha ido adaptando a las necesidades de la industria audiovisual europea y ha intentado dar respuesta a sus necesidades con el objetivo último de favorecer la creación de un mercado audiovisual común dentro de las fronteras de la UE.

\section{Los orígenes y las dos primeras ediciones del programa:}

MEDIA I y MEDIA II

La primera edición del programa MEDIA se pone en marcha para el quinquenio 1991-1995, tras un periodo de toma de decisiones y de pruebas piloto que se inició en 1986, cuando la CE aprobó un programa de acción a favor de la producción audiovisual europea, tras la voluntad manifestada por el Consejo de la Unión Europea (CUE). ${ }^{9}$ El MEDIA I, dotado de un presupuesto de 230 millones de ecus, estableció sus líneas de acción en la fase anterior y posterior de la producción audiovisual, ${ }^{10}$ así como en los diferentes procesos llevados a cabo a lo largo de toda la cadena industrial: formación, preproducción, multilingüismo de los programas (doblaje y subtitulado), utilización de nuevas tecnologías, mecanismos de distribución y promoción comercial, creación del "segundo mercado" a partir de la explotación de archivos y mejora del marco de financiación del sector industrial. El programa MEDIA I tenía como objetivo esencial mejorar la industria a nivel europeo reforzando las condiciones de desarrollo en todos los estadios del sector, desde la formación de los profesionales hasta la promoción de las obras en el mercado único. Además pretendía contribuir a la creación de un espacio audiovisual europeo, a conseguir el equilibrio entre las fuerzas del mercado, a movilizar la financiación en el mercado de la industria de programas y a un enfoque global de la actividad comunitaria a favor del ámbito audiovisual. Se establecieron

9 El CUE marca la dirección política general de la UE, pero no tiene poder para aprobar leyes. Se reúne durante dos o tres días cada seis meses como mínimo, con su presidente a la cabeza, y está compuesto por los jefes de Estado o de gobierno nacionales, además del presidente de la CE.

10 El apoyo a la fase de la producción audiovisual se dejaba en manos de los programas de ayuda a nivel nacional de cada Estado miembro. 
cinco líneas de trabajo (distribución, explotación y promoción, mejora de las condiciones de producción, financiación y formación) a través de 19 proyectos, en los que participaron casi 20 mil sociedades e instituciones y con un funcionamiento descentralizado. El MEDIA I se inició con 12 países miembros y al finalizar contaba con 18 participantes.

En 1995, se aprobó una nueva edición del programa: MEDIA II, con un presupuesto inicial de 400 millones de ecus y una duración hasta el año 2000. Tras la evaluación del primer programa, ${ }^{11}$ se decidió reducir las líneas de actuación y limitarlas a tres grandes ejes de acción: la formación, el desarrollo del producto y la distribución. La finalidad del MEDIA II era conseguir resultados más efectivos en la estructuración de la industria audiovisual europea, evitando la dispersión de efectos que causaron las 19 líneas de actuación del MEDIA I. El presupuesto final fue de 310 millones de ecus, de los cuales 122 millones se destinaron a la distribución cinematográfica (video y salas), 60 millones al desarrollo de la industria, 45 millones a la formación, 17.5 millones a la promoción y 17.5 millones a la distribución en televisión. Los objetivos del programa eran contribuir a la competitividad de la industria audiovisual europea en el mercado, mediante el apoyo al desarrollo de proyectos con un auténtico potencial de difusión; al respeto de la diversidad lingüística y cultural europea; a la valorización del patrimonio audiovisual europeo; al desarrollo de las potencialidades de los países o regiones con escasa capacidad de producción audiovisual y/o con una área lingüística reducida; al desarrollo de un sector de producción y distribución independientes y, en particular, de las pequeñas y medianas empresas.

El programa MEDIA II dio respuesta a muchas de las peticiones que se habían hecho desde el sector profesional, tanto por parte de los autores del informe sobre el audiovisual, como a las conclusiones de la Conferencia Europea del Audiovisual de 1994. Al marcarse solamente

11 Todos los programas europeos tienen una fase final de evaluación y de cada edición del programa MEDIA se ha realizado un informe evaluativo. Del MEDIA I, se identificaron las siguientes debilidades: el nivel de costes operativos con relación a las cantidades manejadas, la multiplicación de iniciativas descoordinadas, la falta de transparencia en el proceso, la falta de control por parte de la CE y la dispersión de las actividades asignadas. 
tres grandes ejes de actuación, tenía más posibilidades de establecer unos fundamentos sólidos para el desarrollo de una industria audiovisual verdaderamente fuerte y competitiva. Así mismo, el MEDIA II se distingue del anterior por las siguientes particularidades: un aumento de $55 \%$ del presupuesto, una centralización de las actividades en tres sectores prioritarios, una mayor transparencia en la gestión de los fondos y una bajada significativa de los gastos generales administrativos. Se modifica también el sistema de ayudas a través de préstamos y/o subvenciones entre 50\% y 75\% del coste total. Además en 1995 se crearon lo que se conocen como los MEDIA Desk y MEDIA Antenae, unas oficinas del programa en cada uno de los estados miembros para ayudar a las empresas audiovisuales en el proceso de información y solicitud.

En esta segunda edición ya se empezaron a constatar una serie de particularidades que caracterizaran al programa MEDIA: su capacidad de impacto económico en el conjunto de la industria audiovisual europea es claramente pequeña, situándose en $0.51 \%$. Respecto de la asignación de recursos, se observa que los cinco grandes países (Alemania, España, Francia, Inglaterra e Italia) consiguen dos tercios de las ayudas del programa (66.26\%), mientras que el resto de participantes se quedan con un tercio (33.74\%) (Bureau d'Information et Prospective Économique (BIPE), 2001).

La segunda década de programa (2001-2011): MEDIA Plus/MEDIA Formación y MEDIA 2007-2013

El programa MEDIA Plus se desarrolló entre 2001 y 2005 con una dotación presupuestaria de 400 millones de euros. Sus líneas de actuación siguieron las establecidas en el programa anterior: ayudas a la distribución, desarrollo y promoción (350 millones) y ayudas a la formación (50 millones). Su aprobación suponía una continuidad en los ejes estratégicos establecidos en la edición anterior. Para reforzar la financiación del sector audiovisual, se aprobó, dando respuesta a las demandas del sector profesional, la iniciativa "Audiovisual i2i" en colaboración con el Banco Europeo de Inversiones. 12

12 El Banco Europeo de Inversiones financia proyectos de inversión de la UE y ayuda a las pequeñas empresas a través del Fondo Europeo de Inversiones. 
La acción de los dos programas, MEDIA Plus y MEDIA Formación, fijada inicialmente para funcionar hasta finales de 2005, se prolongó por un año más hasta diciembre de 2006, a petición del Parlamento Europeo y el CUE. El motivo era poder seguir funcionando frente a la ampliación de la UE a diez nuevos estados miembros en 2004.13 Como consecuencia, se amplió el presupuesto inicial desde 350 hasta 453.6 millones de euros. Las líneas de acción eran: formación de profesionales de la industria, desarrollo de proyectos de producción, distribución de obras cinematográficas y programas audiovisuales, promoción de obras cinematográficas y programas audiovisuales y apoyo a festivales de cine y para la promoción en terceros países. En total se financiaron 9 mil proyectos, de los cuales $21 \%$ fueron de desarrollo, $67 \%$ de distribución y $8 \%$ de formación. El número de proyectos fue incrementándose año a año: en 2001 fueron 974 y en 2006 fueron mil 926. Respecto de la asignación de proyectos por países, se mantiene la proporción de dos tercios para los cinco grandes mercados, por un tercio para el resto de participantes (Euréval, 2007). La evaluación del programa puso de relieve que era necesario modificar principalmente dos aspectos: el acceso al programa a través de una reorganización de los MEDIA Desk, y una mejor coordinación entre las dinámicas administrativas y el ritmo de la industria audiovisual, que suponía principalmente una simplificación de los procesos haciéndolos proporcionales al tipo y tamaño de la subvención. Ambos elementos fueron mejorados en la siguiente edición.

La edición actual del programa MEDIA tiene su periodo de actuación entre 2007 y 2013 con un presupuesto de 755 millones de euros. Comprende una serie de medidas de apoyo a la industria audiovisual europea que se centran en las mismas cinco líneas de actuación de la edición anterior del MEDIA. Esto es, apoyo para la formación de profesionales, el desarrollo de proyectos productivos, distribución de películas y programas audiovisuales, la promoción de películas y programas audiovisuales y el apoyo a festivales de cine. Sus objetivos son reforzar la competitividad del sector audiovisual europeo, en tanto que es reflejo de la identidad

13 Los países que se integraron a la UE en 2004 fueron: Chipre, Eslovaquia, Estonia, Eslovenia, Letonia, Polonia, Hungría, Malta, Lituania y República Checa. 
cultural y del patrimonio europeo; aumentar la circulación de obras audiovisuales europeas dentro y fuera de la UE; fortalecer la competitividad del sector audiovisual europeo, facilitando el acceso a la financiación y la promoción del uso de las tecnologías digitales. Se incorporaron nuevas áreas de actuación: la formación inicial, los trabajos interactivos, el video a la carta, la distribución cinematográfica digital, las comunidades web y los Open Media. En los dos primeros años del programa (2007-2009), se subvencionaron 5 mil proyectos, que fueron solicitados por 3 mil organizaciones y de las cuales, mil 400 recibieron ayudas (58\% a productores, $31 \%$ a distribuidores y $11 \%$ a promotores o exhibidores). En cuanto a la distribución por países, $67 \%$ de los proyectos eran de los cinco grandes mercados; $25 \%$ de los diez países medianos y pequeños, y $8 \%$ para los nuevos países participantes (Euréval, 2010).

Como complemento al programa MEDIA se han creado unos mecanismos complementarios para acceder al préstamo por parte de las pequeñas y medianas empresas audiovisuales europeas. Un instrumento existente es el fondo de garantía "Audiovisual i2i”, que ya fue introducido por el Banco Europeo de Inversiones como complemento de los programas MEDIA Plus y MEDIA Formación (en marcha desde 2002). Su objeto es facilitar el acceso de las empresas de producción europeas a la financiación concedida por los bancos y las instituciones financieras, mediante la cofinanciación de una parte del coste de las partidas siguientes: seguros para producciones audiovisuales, garantía de buen fin para la producción de una obra audiovisual y financiación bancaria para la producción de una obra audiovisual. Desde su creación hasta 2009 , se han financiado 408 proyectos y la financiación total destinada (2002-2010) ha sido de 17 millones 545 mil 521 euros.

En esta misma línea de favorecer el crédito, la CE ha decidido crear otro instrumento para la financiación a las pequeñas y medianas empresas audiovisuales: el fondo MEDIA de garantía para la producción (MEDIA Production Guarantee Fund), enmarcado dentro del programa MEDIA 2007-2013. Tras un estudio realizado durante 2009 para estudiar el papel de los bancos en la financiación cinematográfica, la CE decidió abrir convocatoria (2010/S1-00123) para crear un fondo por un valor 
de 8 millones de euros para un periodo de cuatro años (2010-2013)14 para garantizar el crédito al sector cinematográfico. Esto supondrá un "nuevo" instrumento de apoyo a la industria audiovisual, especialmente ahora que el acceso al crédito es complicado para todos los sectores económicos. Se trata de una contribución comunitaria que se usa como garantía del préstamo cedido por bancos locales a productores cinematográficos. Sin embargo, hay que señalar que este instrumento no es del todo novedoso, porque ya estaba previsto a mediados de los años 1990, como señalan los investigadores Henning y Alpar (2005), pues el Parlamento Europeo ya había dado luz verde a su creación en 1995; es decir, este mecanismo ha visto la luz 15 años después.

Además de estos dos mecanismos, la UE ha puesto en marcha otro instrumento para favorecer los negocios en el sector cultural, y en consecuencia, en el audiovisual, que son los protocolos de cooperación cultural que se incluyen con los acuerdos de libre comercio que firma la UE con otros países y entidades regionales supranacionales. En la actualidad, ya se han suscrito dos que afectan directamente al sector audiovisual: con el CARIFORUM (2008) y con Corea del Sur (2011).

\section{EL BALANCE DE DOS DÉCADAS DE POLÍTICA PÚBLICA DE APOYO}

A LA INDUSTRIA AUDIOVISUAL: APERTURA A LA

INTERNACIONALIZACIÓN Y ESCASO IMPACTO INTERNO

El balance de la evolución del programa MEDIA se puede realizar a partir de dos coordenadas: la externa, dirigida hacia la internacionalización, y la interna, el impacto sobre el mercado audiovisual local. A partir de estos parámetros, será posible evaluar cuál ha sido la evolución de la política pública de apoyo a la industria audiovisual de la UE.

\section{La apertura al mercado internacional}

El programa MEDIA ha tenido desde sus inicios una dimensión exterior. Primero, su voluntad fue la de ampliar el número de países participantes hacia el entorno europeo más próximo y hacia los países candidatos a laUE;

14 Se destinaron 2 millones de euros para la convocatoria de 2010 y los 6 millones restantes son para las tres ediciones posteriores. 
segundo, la importancia creciente del mercado global obligó a la UE a pensar en el mercado global y a iniciar las acciones hacia la internacionalización.

Es a partir de 2006 cuando se empiezan las acciones hacia la ampliación a terceros países fuera del entorno próximo europeo, más allá de lo que se conoce como la política europea de vecindad. De hecho, en el articulado del programa aprobado por el CUE y el Parlamento en 2006 (Decisión No1718/2006/EC), ya aparecen una serie de elementos que establecen la base para esta ampliación a terceros países. En concreto, en dos artículos (5 y 6) se menciona la importancia de los mercados exteriores y en especial, en el artículo 8 se establece la posibilidad de realizar acciones con terceros países. De hecho esta es la base a partir de la cual se fundamenta la acción preparatoria MEDIA International y el programa MEDIA Mundus. Además de estos elementos, otros factores también han ayudado a iniciar la actuación internacional. Por un lado, el informe de evaluación del programa MEDIA Plus ya identificó la importancia de los mercados internacionales para solucionar los problemas de la industria audiovisual europea. Por otro, la propia ampliación de la UE, especialmente con los países que entraron en 2004, que se adhirieron con anterioridad al programa MEDIA, ha permitido ir ampliando el perímetro de países donde estaba vigente dicho programa.

La acción preparatoria MEDIA International tuvo por objetivo financiar actividades de cooperación entre los profesionales europeos del sector audiovisual y sus homólogos de terceros países bajo una base de beneficio mutuo. La duración fue de tres ediciones (2008-2010). Al igual tuvo como finalidad alentar la creación de un flujo bidireccional de obras audiovisuales a nivel de mercados internacionales. Este programa sirvió para preparar el camino al nuevo programa MEDIA Mundus, que se activó en 2011 hasta 2013. El presupuesto fue de 8 millones de euros, distribuidos en tres ediciones tal y como nos presenta la Tabla 2. Es una muestra clara de la voluntad de la CE de dar mayor protagonismo a la dimensión exterior, sobre todo teniendo en cuenta que en el mercado global el audiovisual europeo puede encontrar nuevos aliados y otras vías de comercialización que el mercado interno europeo no le está facilitando. De entre los proyectos concedidos, se observa una gran diversidad de terceros países. Hay que mencionar que en las tres 


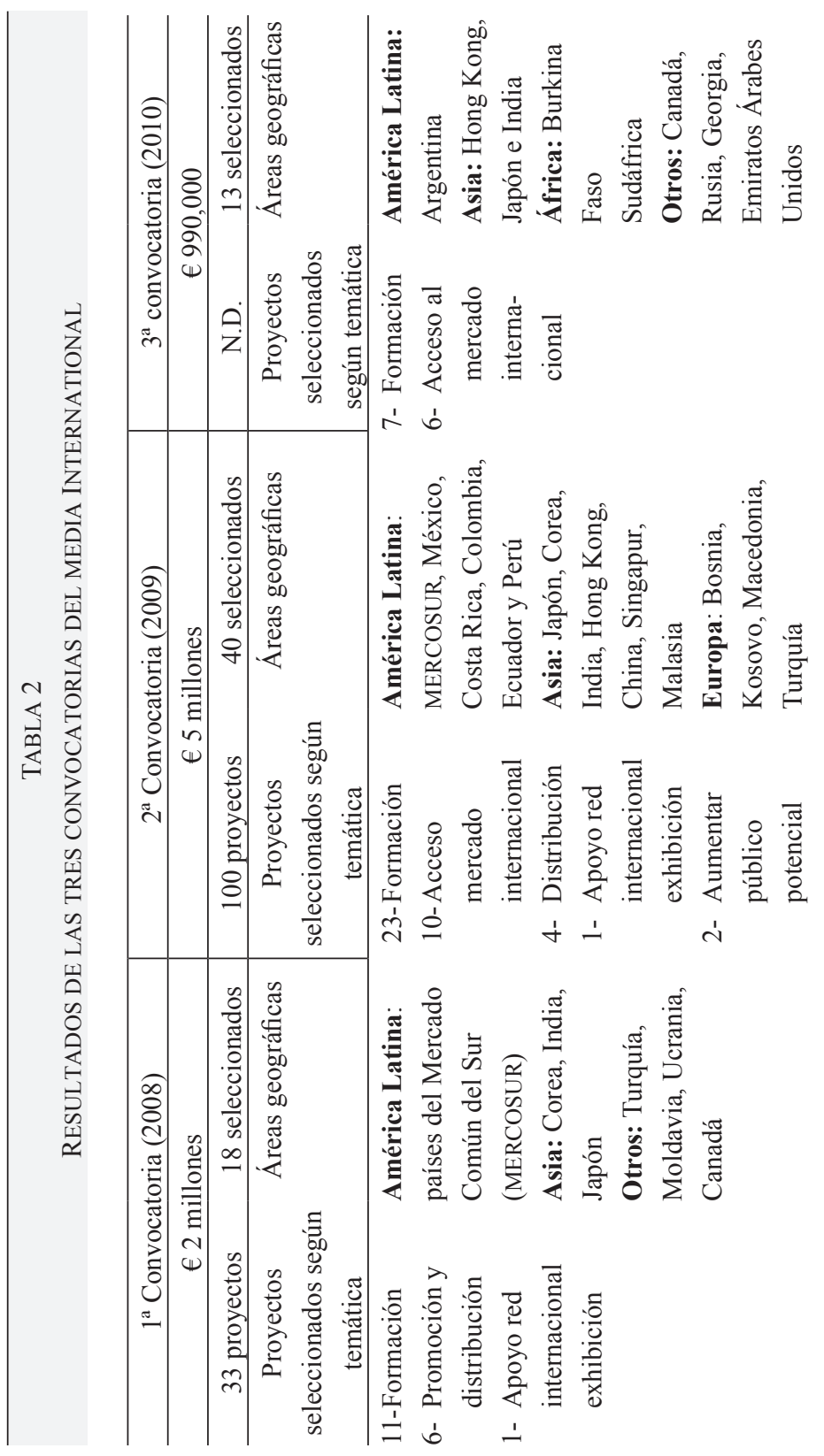




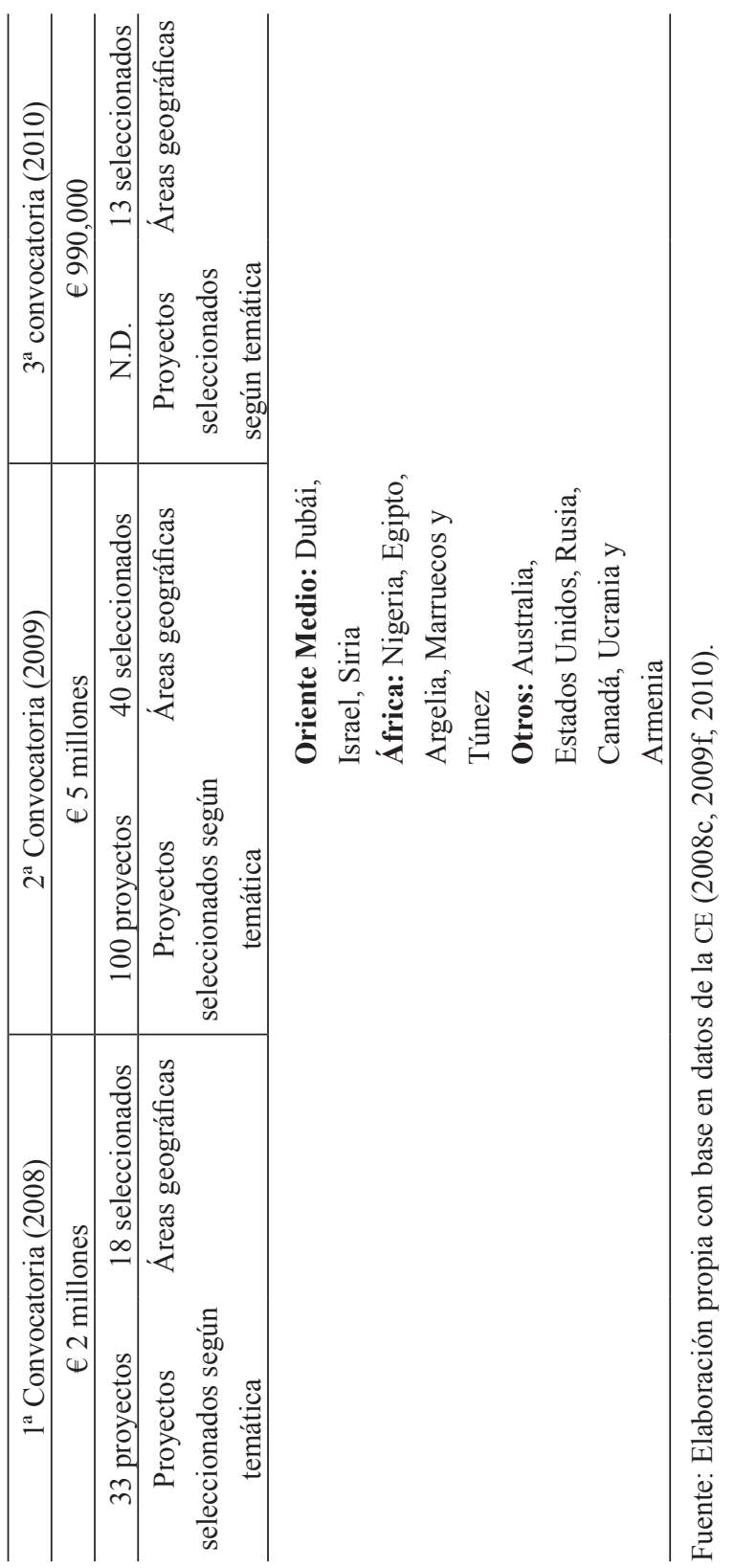


convocatorias participaron países latinoamericanos, en especial, los integrantes del MERCOSUR.

Siguiendo con esta idea de ampliación global, a principios de 2009 se presentó el nuevo programa MEDIA Mundus, con un presupuesto de 15 millones de euros (2011-2013), para la cooperación con profesionales audiovisuales de terceros países. Sus objetivos se centran principalmente en mejorar: a) el intercambio de información entre profesionales europeos y de terceros países, b) la competitividad y la distribución transnacional de las obras europeas, c) la circulación y exposición de las obras europeas a nivel mundial, y d) incrementar la demanda del público en contenido audiovisual culturalmente diverso (Parlamento Europeo \& CUE, 2009). El programa MEDIA Mundus se concibe como una iniciativa para incrementar la competitividad del sector audiovisual en el mercado global y contribuir a la implementación de la Convención de la Organización de las Naciones Unidas para la Educación, la Ciencia y la Cultura (UNESCO) sobre la promoción y protección de la diversidad de las expresiones culturales. También se apunta la posibilidad de incrementar las opciones del consumidor y la diversidad cultural en los mercados europeos. Una particularidad de este programa y del interés que ha tenido por parte de las instituciones comunitarias está en su proceso de aprobación que ha sido realmente rápido, quedando aprobado el programa bajo la decisión 1041/2009/EC el 21 de octubre de 2009, en menos de un año, cuando procesos similares en el ámbito del audiovisual europeo duran una media de tres años. En cuanto a resultados, en la primera convocatoria del MEDIA Mundus (2011), se han concedido un total de 36 proyectos, de los cuales 16 son de formación (1.23 millones de euros); 4 de acceso al mercado (325 mil 750 euros); 3 proyectos de distribución y circulación (665 mil euros); 11 proyectos combinados (1.34 millones de euros), y 2 proyectos complementarios (1.2 millones de euros).

Como complemento a la acción exterior, el programa MEDIA mantiene también una línea de cooperación con otros países. Como muestra la Tabla 3, entre ellos se encuentran países candidatos a ser miembros de la UE y otros que por razones de vecindad mantienen una relación estrecha con la misma. 
TABLA 3

COOPERACIÓN DEL PROGRAMA MEDIA CON TERCEROS PAÍSES

\begin{tabular}{lcc}
\hline Tipología de país & País & Estatus en el programa \\
\hline Países candidatos & Croacia & Miembro de pleno derecho \\
a la adhesión a la & \\
UE bajo el acuerdo & \\
marco &
\end{tabular}

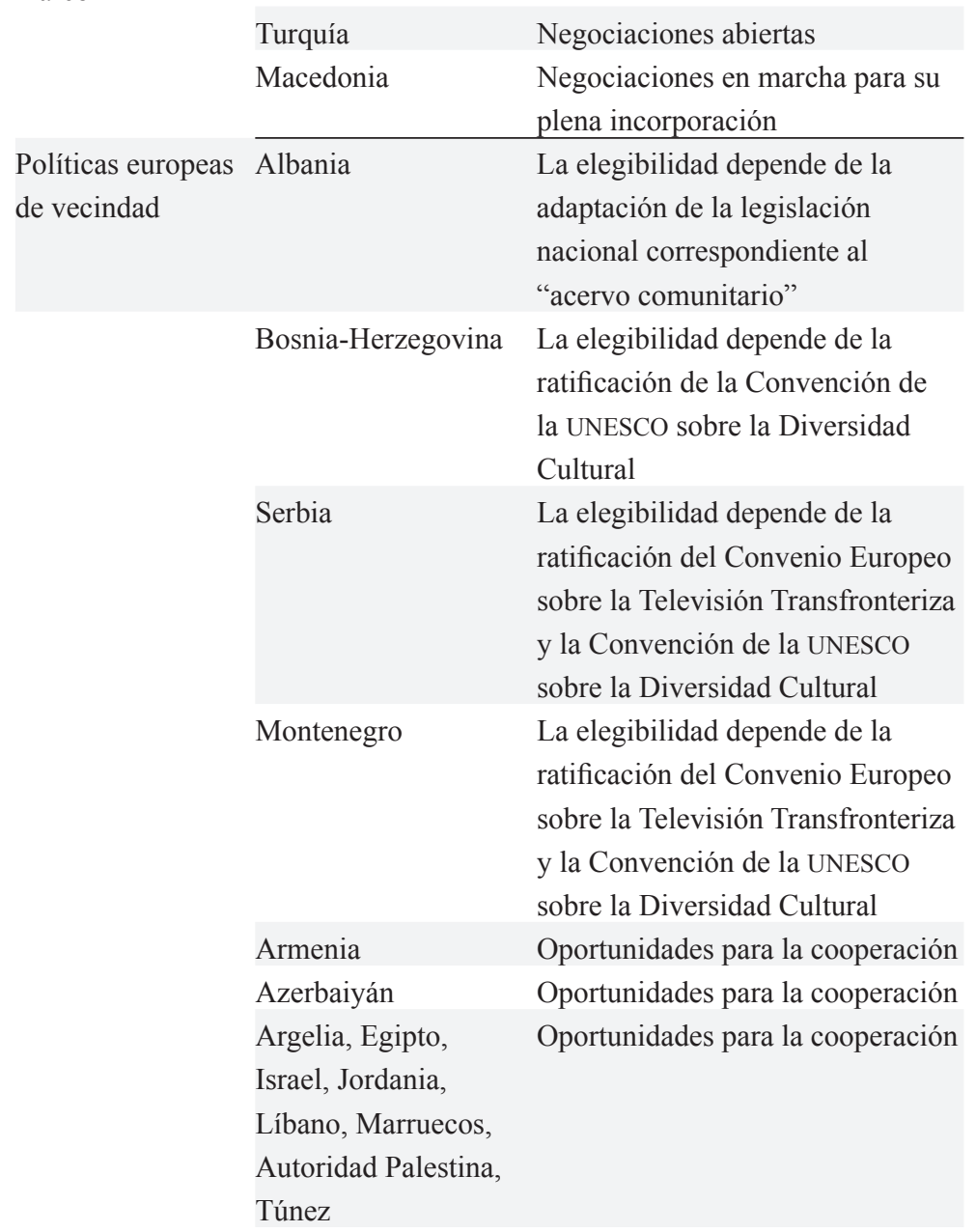




\begin{tabular}{|c|c|c|}
\hline Tipología de país & País & Estatus en el programa \\
\hline & Moldavia & $\begin{array}{l}\text { La elegibilidad depende de la } \\
\text { adaptación de la legislación } \\
\text { nacional correspondiente al } \\
\text { "acervo comunitario" }\end{array}$ \\
\hline & Georgia & Oportunidades para la cooperación \\
\hline & Ucrania & $\begin{array}{l}\text { La elegibilidad depende de las } \\
\text { condiciones establecidas en el } \\
\text { Programa }\end{array}$ \\
\hline & Rusia & $\begin{array}{l}\text { La elegibilidad depende de las } \\
\text { condiciones establecidas en el } \\
\text { Programa }\end{array}$ \\
\hline
\end{tabular}

Fuente: CE (2011).

\section{Impacto sobre el mercado audiovisual europeo}

El principal objetivo del programa MEDIA es consolidar el mercado audiovisual interno, denominado en la jerga comunitaria como el espacio audiovisual europeo, para fortalecer a la industria europea y favorecer la circulación de las obras europeas. Para evaluar el funcionamiento del programa, ${ }^{15}$ se tomarán dos datos que ilustran cómo ha evolucionado el programa y el mercado europeo: por un lado, la relación entre la producción cinematográfica y la cuota de mercado del cine europeo, y por otro, la participación de los distintos estados miembros en el programa. Ambos elementos nos permiten analizar el impacto en el mercado interno.

Si tomamos como referencia la producción cinematográfica europea, observamos, como nos muestra la Figura 1, que el incremento de obras cinematográficas ha sido constante en la última década (20002010), de hecho ha sido muy superior al de otras industrias como la estadounidense, llegando a superar las mil producciones.

15 La CE ha realizado una evaluación del programa MEDIA en cada una de sus ediciones. Es a partir del estudio detallado de cada uno de ellos que se ha elaborado este epígrafe (CE, s.f.-c). 
FIGURA 1

EVOLUCIÓN DE LA PRODUCCIÓN CINEMATOGRÁFICA EN LA UE

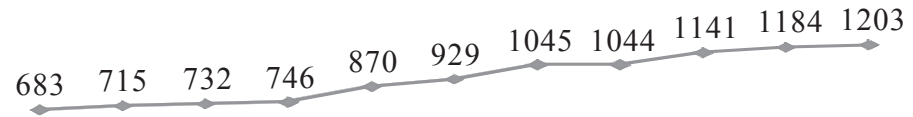

20002001200220032004200520062007200820092010

Fuente: Elaboración propia con base en datos del Observatorio Audiovisual Europeo (OAE), 2011.

Si junto a los datos de producción ponemos en relación los datos de la cuota de mercado del cine en función de su nacionalidad (véase Tabla 4), entonces nos damos cuenta que la cuota de mercado de las producciones europeas disminuye en las salas de cine a pesar de que la producción aumenta considerablemente (véase Figura 1) y no sufre modificación de la tendencia a pesar de que en 2004 se produce la ampliación de la UE con la entrada de diez países del antiguo bloque de países de la Europa del Este. La cifra europea se mantiene en torno a $25 \%$, con oscilaciones por encima o por debajo según el año

\section{TABLA 4}

PORCENTAJES DE DISTRIBUCIÓN DEL MERCADO EUROPEO POR ORIGEN DE LAS PRODUCCIONES (2000-2010)

\begin{tabular}{lrrrrrrrrrr}
\hline & 2001 & 2002 & 2003 & 2004 & 2005 & 2006 & 2007 & 2008 & 2009 & 2010 \\
\hline Cine nacional & 21.8 & 19.5 & 19.4 & & & & & & & \\
Cine europeo & 9.3 & 7.9 & 6.3 & & & & & & & \\
que circula & & & & & & & & & & \\
Total cine & 31.1 & 27.4 & 25.7 & 26.5 & 24.6 & 27.9 & 28.1 & 28.3 & 26.8 & 25.3 \\
europeo & & & & & & & & & & \\
Coprod. & & & & & 13.3 & 5.6 & 7.5 & 4.4 & 4.0 & 5.4 \\
EU-EE.UU. & & & & & & & & & & \\
Cine EE.UU. & 65.4 & 71.2 & 72.2 & 71.4 & 59.9 & 63.4 & 62.6 & 65.5 & 66.9 & 68.0 \\
Otros & 3.5 & 1.3 & 2.2 & 2.1 & 2.2 & 3.2 & 1.8 & 1.8 & 2.3 & 1.3 \\
\hline
\end{tabular}

Fuente: Elaboración propia con base en datos del OAE $(2010,2011)$ 
Las cifras sobre la circulación interna de las obras europeas no se encuentran disponibles a partir de 2003. La principal fuente estadística europea, el OAE, ha dejado de publicar esta información. Pero los datos que existen muestran que el espacio audiovisual europeo tampoco funciona para las producciones europeas, para quienes sí funciona es para las obras estadounidenses que tienen la mayor cuota de mercado en todos los países de la UE, situación similar a la que se vive en muchos países latinoamericanos. Además, el dato de las coproducciones EU-EE.UU. corresponde a productos realizados en su mayoría con empresas británicas. Estas coproducciones favorecen que posteriormente puedan contabilizarse como europeas y se incorporen al cómputo necesario para el cumplimiento de las cuotas de difusión de obras de origen europeo en todas las televisiones de la UE, como establece la Directiva de Servicios de Comunicación Audiovisual. Es decir, estas coproducciones en su mayoría británico-estadounidenses se benefician de los instrumentos proteccionistas de la política audiovisual europea, cuando estas cuotas son objeto de crítica constante por parte de la industria de Hollywood y en especial, de su lobby, la Motion Pictures Association of America (MPAA).

$\mathrm{Si}$ analizamos los resultados de las asignaciones de los recursos de las distintas convocatorias del programa MEDIA (véase Tabla 5), observamos que se refuerza la idea de que el programa ayuda a las cinco grandes industrias europeas (Alemania, España, Francia, Inglaterra e Italia), que consiguen dos tercios de la ayuda, mientras que los países pequeños quedan en posiciones posteriores y obtienen solo un tercio de los recursos. Este predominio de los cinco grandes países también se produce en el otro programa europeo de referencia de ayuda a la industria audiovisual, el EURIMAGES, del Consejo de Europa16 (De Vinck, 2009). Este dato puede tener una doble lectura: por un lado, fortalece

16 El CE, con sede en Estrasburgo (Francia), cuenta con 47 países miembros, cubriendo casi la totalidad del continente europeo. Fundado el 5 de mayo de 1949 por diez países, tiene como objetivo desarrollar los principios europeos comunes y democráticos basados en el Convenio Europeo de Derechos Humanos y otros textos de referencia sobre la protección de las personas. 
la industria de los cinco grandes países, fundamental para poder construir una industria europea fuerte fuera de sus mercados de origen, y por otro, si el objetivo es fomentar una mayor proporción de obras de otros países fuera de los grandes, entonces la tendencia es que sigue manteniéndose en una minoría (aunque hay que apuntar que en algunos estados miembros existe poca tradición de producción audiovisual).

TABLA 5

CLASIFICACIÓN DE LOS PAÍSES EN FUNCIÓN DE LOS RECURSOS OBTENIDOS DEL PROGRAMA MEDIA POR CONVOCATORIAS

\begin{tabular}{|c|c|c|c|c|c|}
\hline País & $\begin{array}{c}\text { MEDIA } \\
2006 \\
\end{array}$ & $\begin{array}{c}\text { MEDIA } \\
2007 \\
\end{array}$ & $\begin{array}{c}\text { MEDIA } \\
2008 \\
\end{array}$ & $\begin{array}{c}\text { MEDIA } \\
2009 \\
\end{array}$ & $\begin{array}{c}\text { MEDIA } \\
2010 \\
\end{array}$ \\
\hline Francia & $1^{\circ}$ & $1^{\circ}$ & $1^{\circ}$ & $1^{\circ}$ & $1^{\circ}$ \\
\hline Alemania & $2^{\circ}$ & $2^{\circ}$ & $2^{\circ}$ & $2^{\circ}$ & $2^{\circ}$ \\
\hline Italia & $3^{\circ}$ & $3^{\circ}$ & $3^{\circ}$ & $3^{\circ}$ & $4^{\circ}$ \\
\hline España & $4^{\circ}$ & $5^{\circ}$ & $4^{o}$ & $4^{\circ}$ & $5^{\circ}$ \\
\hline Inglaterra & $5^{\circ}$ & $4^{\circ}$ & $5^{\circ}$ & $5^{\circ}$ & $3^{\circ}$ \\
\hline Bélgica & $6^{\circ}$ & $6^{0}$ & $6^{\circ}$ & $6^{\circ}$ & $6^{\circ}$ \\
\hline Holanda & $7^{\circ}$ & $7^{\circ}$ & $7^{\circ}$ & $7^{\circ}$ & $7^{\circ}$ \\
\hline Suecia & $8^{\circ}$ & $10^{\circ}$ & $8^{\circ}$ & $8^{\circ}$ & $9^{\circ}$ \\
\hline Dinamarca & $10^{\circ}$ & $8^{\circ}$ & $11^{\circ}$ & $11^{\circ}$ & $8^{\circ}$ \\
\hline Suiza & $9^{\circ}$ & $9^{\circ}$ & $9^{\circ}$ & $9^{\circ}$ & $11^{\circ}$ \\
\hline Polonia & $11^{\circ}$ & $11^{\circ}$ & $10^{\circ}$ & $10^{\circ}$ & $10^{\circ}$ \\
\hline
\end{tabular}

Fuente: Elaboración propia con base en datos de MEDIA Antena Catalunya (2006, 2007, 2008, 2009, 2010).

Otro aspecto a remarcar es la dotación presupuestaria del programa MEDIA. Si bien es cierto que en estas dos décadas ha triplicado su presupuesto, su montante ha sido el resultado de negociación entre los estados miembros que mantienen una división interna sobre los recursos a asignar al programa. Mientras que su gran defensor es Francia; Inglaterra y Alemania, por su tradición liberal en la concepción de la política europea, se erigen como los más reticentes a aumentar el presupuesto comunitario en este ámbito (Humphreys, 2010). Debido a este posicionamiento, el presupuesto dedicado al MEDIA tiene una capacidad de 
impacto realmente mínima en el conjunto de la industria audiovisual europea. En este sentido, es necesario aportar otros datos que puedan ayudar a evaluar si se trata de cantidades relevantes dentro del contexto comunitario. Por un lado, una cifra significativa es el presupuesto anual que tiene el Centre National de la Cinematographie de Francia, cuyo mercado audiovisual es referencia en el seno de la UE, el cual contaba con recursos por valor de 749 millones de euros para 2011, cantidad que supone el mismo presupuesto que el programa MEDIA para 32 países y para un periodo de siete años; por lo que las ayudas francesas ponen de relieve la poca magnitud desde un punto de vista global de apoyo a la industria audiovisual europea. Por otro, la cifra de 100 millones de euros anuales para la política de ayudas a la industria audiovisual parece irrisoria teniendo en cuenta las cifras que manejan otros programas europeos, como es el caso de la parte de recursos del séptimo programa marco (principal programa de investigación) destinada al desarrollo de la sociedad de la información, que se eleva a 1,800 millones de euros anuales. Estas cifras de las ayudas al audiovisual muestran una realidad constante en la historia de la política audiovisual europea: un desequilibrio entre la importancia estratégica que se otorga al audiovisual en los documentos y discursos oficiales y los escasos recursos finalmente destinados a financiar este sector, quedando en evidencia que ciertamente el audiovisual no se encuentra entre las prioridades de la agenda estratégica presupuestaria comunitaria.

\section{EL FUTURO DEL PROGRAMA MEDIA: EL HORIZONTE 2020}

Con el objetivo de adaptar el programa MEDIA a escenarios futuros, CE ya está planificando las próximas acciones comunitarias con vistas al horizonte 2020. Su idea es integrar los distintos programas de cultura y las diferentes modalidades del programa MEDIA en un nuevo programa, que se llamará Creative Europe. Sus objetivos estarán acordes a los del proyecto Europa 2020, con la finalidad principal de crear puestos de trabajo en los sectores cultural y creativo. Su acción pretende impulsar la acción más allá de las acciones nacionales y establecer un fuerte vínculo con la promoción de la diversidad lingüística y cultural. Así mismo se contempla el uso de instrumentos financieros innovadores para dar 
apoyo a las industrias. La gestión del programa dependerá de la Agencia Ejecutiva de Educación, Audiovisual y Comunicación, que ya está a cargo en la actualidad de Cultura y de MEDIA. El instrumento financiero previsto estará desarrollado por el Banco Europeo de Inversiones para proveer financiación de deuda y capital para las industrias culturales y creativas. El presupuesto inicial que se propone para el periodo 20142020 es de 1,600 millones de euros. Esta propuesta pone de relieve un primer cambio: la disolución de la particularidad del audiovisual para integrarlo dentro de la categoría de industrias creativas, un concepto que no está exento de un carácter marcadamente mercantilista que tiende a minimizar el papel de la cultura en favor de elementos como la creatividad y la innovación (Bustamante, 2011).

Otro dato interesante a señalar es la dotación presupuestaria que se dobla respecto del programa anterior, pero hay que tener en cuenta que se agruparán no solo las acciones en el ámbito audiovisual sino también en el área de la cultura. Seguiremos en este sentido en la misma línea de voluntarismo europeo, pero con capacidad de poco impacto, mínima sobre el conjunto del sector audiovisual teniendo en cuenta el escaso volumen de las ayudas del programa MEDIA. Cierto es que estas ayudas se pueden sumar a las de ámbito estatal en virtud del principio de subsidiaridad, pero en términos globales si pretende fortalecer a la industria europea y la diversidad cultural con este volumen de apoyo difícilmente se podrá obtener impacto. También hay que señalar que, como indican De Vinck, Biltereyst y Pauwels (2007):

El enfoque de la UE está demasiado centrado en la oferta, esperando que la demanda llegue automáticamente. Sin embargo, las cosas no son tan simples, y las actuales trasposiciones nacionales de las políticas europeas no suponen automáticamente una oferta culturalmente diversa, ni tampoco el fortalecimiento de la identidad europea con estas acciones (p. 59).

En esta misma línea, Anna Herold (2004) también indica que "la Unión Europea ya admite que la creación de un mercado común para películas no puede garantizar por sí misma el pluralismo del contenido cultural" (p. 17), aunque de momento sigue esta misma norma de actuación. 
Con relación a la dimensión internacional del programa MEDIA habrá que esperar a conocer los detalles sobre el futuro programa de las industrias creativas y si se incluye la modalidad de cooperación con terceros países. Así mismo está en cuestión la estrategia de la UE de consolidarse como un actor relevante en el escenario internacional, en la línea que indica el Tratado de Lisboa. Este deseo de liderazgo mundial se le une el objetivo de la UE de convertirse en el gran promotor y defensor de la Convención UNESCO sobre la Diversidad Cultural en el ámbito mundial. Ambos deseos se encuentran en una encrucijada por dos motivos fundamentales: la crisis económica en la que está sumida gran parte de los países miembros de la UE que obliga a dedicar los esfuerzos comunitarios a rescatar las economías de algunos países miembros (como Grecia y Portugal), y la parálisis que sufre la UNESCO por la retirada de Estados Unidos al aceptar como nuevo miembro a Palestina y como consecuencia, los efectos que puede tener en la promoción y defensa de la Diversidad Cultural.

En definitiva, el programa MEDIA se ha convertido en el gran instrumento de la política pública audiovisual europea durante dos décadas. Sus resultados son valorados positivamente si se tienen en cuenta los recursos de los que se disponen, que no siempre están con relación a los objetivos marcados porque su capacidad de impacto sobre el sector es realmente testimonial. Su singularidad se encuentra en cuestión en el escenario más próximo, ya que las intenciones de la $\mathrm{CE}$ apuntan hacia diluir su especificidad dentro de un programa contenedor bajo el paraguas de las industrias creativas. Habrá que esperar a ver cómo reaccionan los países miembros más activamente defensores del audiovisual, liderados por Francia, y el conjunto de profesionales europeos de la industria audiovisual.

\section{Bibliografía}

Bureau d'Information et Prospective Économique-BIPE. (2001). Final evaluation of the MEDIA II Programme. Recuperado el 1 de septiembre de $2011 \mathrm{de}$ http://ec.europa.eu/culture/media/programme/docs/ overview/evaluation/reports/rmed2_en.pdf

Bustamante, E. (Coord.). (2011). Las industrias creativas. Amenazas sobre la cultura digital. Barcelona, España: Gedisa. 
Comisión Europea-CE. (2008a). Audiovisual Cooperation Programme "MEDIA MUNDUS". Summary of the results of the public consultation. Recuperado el 20 de junio de 2009 de http://ec.europa.eu/culture/media/media-content/media-mundus/pc.pdf

Comisión Europea-CE. (2008b). Cannes Declaration/Declaration of European Audiovisual Affairs Ministers and of the European Commission member responsible for the Information Society and Media, Europe Day at the Cannes Festival. Recuperado el 20 de junio de 2009 de http://ec.europa.eu/avpolicy/docs/ext/cannes_2008_en.pdf

Comisión Europea-CE. (2008c). MEDIA INTERNATIONAL. Recuperado el 10 de octubre de 2011 de http://ec.europa.eu/culture/media/ media-mundus/international_results_2008_en.htm

Comisión Europea-CE. (2009a). El cine europeo se mundializa: 40 proyectos internacionales de cooperación cinematográfica reciben ayudas comunitarias por un importe de 5 millones de euros, IP/09/1334. Recuperado el 2 de diciembre de 2009 de http://europa. $\mathrm{eu} / \mathrm{rapid} /$ pressReleasesAction.do? reference $=\mathrm{IP} / 09 / 1334 \&$ format $=\mathrm{H}$ TML\&aged $=0$ \&language $=\mathrm{EN}$

Comisión Europea-CE. (2009b). External Dimension of Audiovisual Policy (Staff Working Document), SEC (2009) 1033 final. Recuperado el 30 de julio de 2009 de http://ec.europa.eu/avpolicy/docs/ext/ external_swp.pdf

Comisión Europea-CE. (2009c). Proposal for a Decision of The European Parliament and of the Council establishing an audiovisual cooperation programme with professionals from third countries MEDIA Mundus (COM (2008) 892 final). Recuperado el 1 de septiembre de 2009 de http://ec.europa.eu/information_society/media/docs/ mundus/mundus_proposal_en.pdf

Comisión Europea-CE. (2009d). Media Mundus. Summary of the Impact Assessment, SEC (2008) 3097 final. Recuperado el 20 de junio de 2009 de http://ec.europa.eu/information_society/media/docs/ mundus/mm_ria/en.pdf

Comisión Europea-CE. (2009e). Cinema: EU film support goes global, IP/09/26. Recuperado el 20 de junio de 2009 de http://europa.eu/ $\mathrm{rapid} /$ pressReleasesAction.do?reference $=\mathrm{IP} / 09 / 26 \&$ format $=\mathrm{HTML}$ \&aged $=0$ \&language $=\mathrm{EN} \&$ guiLanguage $=\mathrm{en}$ 
Comisión Europea-CE. (2009f). Convocatoria programa MEDIA INTERNATIONAL. Recuperado el 10 de octubre de 2011 de http://ec.europa. $\mathrm{eu} / \mathrm{culture} / \mathrm{media} / \mathrm{media}-\mathrm{mundus} /$ international_results_2009_en.htm

Comisión Europea-CE. (2010). Convocatoria programa MEDIA INTERNATIONAL. Recuperado el 10 de octubre de 2011 de http://ec.europa. eu/culture/media/media-mundus/international_results_2010_en.htm

ComisiónEuropea-CE.(2011). Paísesmiembros delprogramaMEDIA.Recuperadoel10deoctubrede2011 dehttp://ec.europa.eu/culture/media/ about/media_near_you_en.htm

Comisión Europea-CE. (s.f.-a). Europa 2020. Recuperado el 10 de octubre de 2011 de http://ec.europea.ue/europe2020/index_es.htm

Comisión Europea-CE. (s.f.-b). MEDIA Programme. Recuperado el 10 de octubre de 2011 de http://ec.europea.eu/culture/media/index_en.html

Comisión Europea-CE. (s.f.-c). MEDIA Programme. Recuperado el 10 de octubre de 2011 de http://ec.europa.eu/culture/media/programme/overview/evaluation/reports/index_en.htm

Consejo de la Unión Europea-CUE. (2007, 31 de octubre). Draft Council Resolution on a European agenda for culture - Adoption of the Resolution, 14485/07. Recuperado el 20 de junio de 2009 de http:// www.cultureactioneurope.org/images/stories/advocatepdf/eucounci lresolutioneuagendaforculture31.10.07en.pdf?e4b73c3745ac4bc37 $4714928 \mathrm{e} 835769 \mathrm{~b}=163 \mathrm{~d} 48973 \mathrm{a} 71 \mathrm{~d} 05 \mathrm{dc} 42 \mathrm{ea} 8 \mathrm{e} 8 \mathrm{bfeae} 4 \mathrm{fc}$

Consejo de la Unión Europea-CUE. (2008, 20 de noviembre). Council Conclusions on the promotion of cultural diversity and intercultural dialogue in the external relations of the Union and its Member States, 2905th Education, Youth and Culture Council meeting. Recuperado el 20 junio de 2009 de http://ec.europa.eu/culture/ourpolicydevelopment/doc/ICD_external_relations_en.doc.pdf

Crusafon, C. (1999). El espacio audiovisual europeo. Análisis de la industria audiovisual y de las políticas europeas en la década de los 90. Tesis doctoral no publicada, Universitat Autònoma de Barcelona, Barcelona, España.

Crusafon, C. (2005). Los desafíos de la política audiovisual europea en la Europa de los 25. Actas del III Congreso Panamericano. Buenos Aires, Argentina: Universidad de Buenos Aires.

Crusafon, C. (2009). Mercado vs. cultura: balance de la Comisión Baroso (2004-2009). Estudos em Comunicaçao, 6, 105-123. 
Crusafon, C. (2010). Audiovisual europeo, diversidad cultural y mercado global: análisis de las actuaciones exteriores de la política audiovisual de la Unión Europea. ÁMBITOS. Revista Internacional de Comunicación, 19, 223-244.

De Bens, E. (Ed.). (2007). Media between Culture and Commerce. Londres, Inglaterra: Intellect.

De Vinck, S. (2009). Europudding or Europaradise? A performing evaluation of the Eurimages co-production film fund, twenty years after its inception. Communications, 34, 257-287.

De Vinck, S., Biltereyst, D. \& Pauwels, C. (2007). Our policies keep on reinventing the past: An overview of EU policy-making in the audiovisual domain. En L. D’Haenens \& F. Saeys (Eds.), Western broadcast models. Structure, Conduct and performance (pp. 25-59). Berlín, Alemania: Mouton de Gruyter.

De Vinck, S. \& Pauwels, C. (2008). Cultural diversity as the final outcome of EU policymaking in the audiovisual sector: A critical analysis. En H. Schneider \& P. Van Den Bossche (Eds.), Protection of cultural diversity from a European and international perspective (pp. 263-316). Amberes/Oxford/Portland: Intersentia.

Euréval (2007, noviembre). Final Evaluation of the MEDIA Plus and MEDIA Training Programmes. Recuperado el 10 de octubre de 2011 de http://ec.europa.eu/culture/media/media-content/documents/ about/evaluations/executive_sum.pdf

Euréval (2010). Évaluation intermediare de MEDIA 2007. Rapport finale-version definitive. Recuperado el 10 de octubre de 2011 de http://ec.europa.eu/dgs/education_culture/evalreports/culture/2010/ media_fr.pdf

Henning, V. \& Alpar, A. (2005). Public aid mechanisms in feature film production: The EU MEDIA Plus Programme. Media, Culture \& Society, 27 (2), 229-250.

Herold, A. (2004). EU Film policy: Between art and commerce. European Diversity and Autonomy Papers EDAP, 3, 5-21.

Humphreys, P. (2003). Regulatory policy and national content. En M. Raboy, (Ed.), L'Avenir de la Réglementation de la Radiodiffusion (pp. 177-207). Québec, Canadá: Centre d’études sur les médiasUniversité Laval. 
Humphreys, P. (2008a). The principal axes of the European Union's Audiovisual Policy. En I. Fernández Alonso y M. Moragas (Eds.), Communication and Cultural Policies in Europe (pp. 151-184). Bellaterra, Barcelona, España: Universitat Autònoma de Barcelona.

Humphreys, P. (2008b). EU Audiovisual Policy, Cultural Diversity and Future of Public Service Broadcating. En J. Harrison \& B. Wessels (Eds.), Mediating Europe. New media and the European poublic sphere (pp. 183-212). Londres, Inglaterra: Berghahn Books.

Humphreys, P. (2010). How deregulatory has the European Union really been in the Audiovisual Sector. Trabajo presentado en la Conferencia de la ICA.

Jakubowicz, K. (2007). Media Governance Structures in Europe. En E. De Bens, (Ed.), Media between Culture and Commerce (pp. 197223). Londres, Inglaterra: Intellect.

Kanzler, M. \& Lange, A. (2008). The place of third country film and audiovisual works in European markets. Estrasburgo, Francia: European Audiovisual Observatory.

Karippinen, K. (2006). Media diversity and the politics of criteria. Diversity assessment and technocratisation of European Media Policy. Nordicom Review, 27 (2), 53-68.

MEDIA Antena Catalunya. (2006, septiembre). MEDIA. Revista de información del Programa MEDIA de la UE. Recuperado el 10 de octubre de 2011 de antenamediacat.com/rcs_auth/convocatories/ setembre_06.pdf

MEDIA Antena Catalunya. (2007, septiembre). MEDIA. Revista de información del Programa MEDIA de la UE. Recuperado el 10 de octubre de 2011 de antenamediacat.com/rcs_auth/convocatories/ setembre_07.pdf

MEDIA Antena Catalunya. (2008, septiembre). MEDIA. Revista de información del Programa MEDIA de la UE. Recuperado el 10 de octubre de 2011 de antenamediacat.com/rcs_auth/convocatories/ setembre_08.pdf

MEDIA Antena Catalunya. (2009, septiembre). MEDIA. Revista de información del Programa MEDIA de la UE. Recuperado el 10 de octubre de 2011 de antenamediacat.com/rcs_auth/convocatories/ setembre_09.pdf 
MEDIA Antena Catalunya. (2010, septiembre). MEDIA. Revista de información del Programa MEDIA de la UE. Recuperado el 10 de octubre de 2011 de antenamediacat.com/res_auth/convocatories/ setembre_10.pdf

Observatorio Audiovisual Europeo-OAE. (2010). Focus 2010. World Film Market Trends. Estrasburgo, Francia: Marché du Film/OAE.

Observatorio Audiovisual Europeo-OAE. (2011). Focus 2011. World Film Market Trends. Estrasburgo, Francia: Marché du Film/OAE.

Parlamento Europeo \& Consejo de la Unión Europea-CUE. (2006, 24 de noviembre). Decisión No. 1718/2006/CE del Parlamento Europeo y del Consejo de 15 de noviembre de 2006 relativa a la aplicación de un programa de apoyo al sector audiovisual europeo (MEDIA 2007). Diario Oficial de la Unión Europea, L327/12. Recuperado el 1 de septiembre de 2009 de http://eurlex.europa.eu/LexUriServ/site/es/ oj/2006/1_327/1_32720061124es00120029.pdf

Parlamento Europeo \& Consejo de la Unión Europea-CUE. (2007, 18 de diciembre). Directiva 2007/65/Ce del Parlamento Europeo y del Consejo de 11 de diciembre de 2007 por la que se modifica la Directiva 89/552/CEE del Consejo sobre la coordinación de determinadas disposiciones legales, reglamentarias y administrativas de los Estados miembros relativas al ejercicio de actividades de radiodifusión televisiva. Diario Oficial de la Unión Europea, L332/27. Recuperado el 1 de septiembre de 2009 de http://eurlex.europa.eu/LexUriServ/LexUriServ.do?uri=OJ:L:2007:332:0027:0045:ES:PDF)

Parlamento Europeo \& Consejo de la Unión Europea-CUE. (2009, 4 de noviembre). Decision No 1041/2009/Ec of the European Parliament and of the Council of 21 October 2009 establishing an audiovisual cooperation programme with professionals from third countries (MEDIA Mundus). Official Journal of the European Union, L288/10. Recuperado el 30 de octubre de 2009 de http://eurlex.europa.eu/ LexUriServ/LexUriServ.do?uri=OJ:L:2009:288:0010:0017:EN:PDF

Pauwels, C., Kalimo, H., Donders, K. \& Van Rompuy, B. (Eds.). (2009). Rethinking European Media and Communications Policy. Bruselas, Bélgica: Brussels University Press. 
Sarikakis, K. (Ed.). (2007). Media and Cultural Policy in the European Union. European Studies-An interdisciplinary series in European culture, history and politics. Amsterdam, Holanda/Nueva York, EE.UU.: Rodopi.

Terzis, G. (Ed.). (2008). European media governance. The Brussels dimension, Bristol, Inglaterra: Intellect.

Fecha de recepción: 07/12/11. Aceptación: 19/02/12. 